\title{
Optical In-Situ Plasma Process Monitoring Technique for Detection of Abnormal Plasma Discharge
}

\author{
Sang Jeen Hong ${ }^{\dagger}$, Jong Hwan Ahn, and Won Taek Park \\ Department of Electronic Engineering, Myongji University, Yongin 449-728, Korea \\ Gary S. May \\ School of Electrical and Computer Engineering, Georgia Institute of Technology, Atlanta 30332, USA
}

Received November 8, 2011; Revised December 13, 2012; Accepted February 4, 2013

\begin{abstract}
Advanced semiconductor manufacturing technology requires methods to maximize tool efficiency and improve product quality by reducing process variability. Real-time plasma process monitoring and diagnosis have become crucial for fault detection and classification (FDC) and advanced process control (APC). Additional sensors may increase the accuracy of detection of process anomalies, and optical monitoring methods are non-invasive. In this paper, we propose the use of a chromatic data acquisition system for real-time in-situ plasma process monitoring called the Plasma Eyes Chromatic System (PECS). The proposed system was initially tested in a six-inch research tool, and it was then further evaluated for its potential to detect process anomalies in an eight-inch production tool for etching blanket oxide films. Chromatic representation of the PECS output shows a clear correlation with small changes in process parameters, such as RF power, pressure, and gas flow. We also present how the PECS may be adapted as an in-situ plasma arc detector. The proposed system can provide useful indications of a faulty process in a timely and non-invasive manner for successful run-to-run (R2R) control and FDC.
\end{abstract}

Keywords: Advanced process control, Plasma process monitoring, Plasma arc detection

\section{INTRODUCTION}

Semiconductor manufacturing operations include raw material supply, manufacturing systems, and finished products. Product applications for electronics are realized by chip manufacturers through industrial standards, production tools, materials and chemicals, metrology, and the technical workforce. Process control in semiconductor manufacturing has emerged as a crucial factor in creating cost-effective electronic manufacturing processes. Starting with statistical process control (SPC) in the 1980s, and continuing with computer integrated manufacturing (CIM) and advanced process control (APC), intelligent manufac-

\footnotetext{
${ }^{\dagger}$ Author to whom all correspondence should be addressed: E-mail: samhong@mju.ac.kr

turing systems (IMS) have consistently improved the quality of semiconductor products, as well as manufacturing effectiveness.

In integrated circuit (IC) manufacturing, the fabrication processes consist of several hundred sequential steps, and the potential yield losses that occur at every step cannot be ignored. The components of process control operations in leading-edge semiconductor manufacturing can be categorized as follows: 1) a run-to-run control basis (lot-to-lot or wafer-to-wafer control) at the lower level; 2) supervised control (tracking process by process) at a higher level; 3) a flexible manufacturing system resulting from powerful CIM concepts; 4) advanced in-situ, post-process, and end-of-the-line metrology; 5) quality control, equipment maintenance, and diagnosis; 6) rapid failure recovery and inventory management; and 7) a virtual manufacturing environment. The ultimate goals of semiconductor manufacturing are minimizing cost, maximizing product quality, reducing variability, improving yield, and extending reliability.

To accomplish reduced process variability, fault detection and classification (FDC) using tool data analysis and APC with in- 
Table 1. Roles of Semiconductor AEC/APC technology sectors.

\begin{tabular}{|c|c|}
\hline Technology Sectors & $\begin{array}{l}\text { Major Role for Contributing } \\
\text { Semiconductor Manufacturing }\end{array}$ \\
\hline $\begin{array}{l}\text { Chip Manufacturing } \\
\text { Sector }\end{array}$ & $\begin{array}{l}\text { Fab-wide infrastructures and distributed } \\
\text { application frameworks. } \\
\text { Multi-purpose hierarchical control appli- } \\
\text { cation using numerous data. }\end{array}$ \\
\hline Framework Sector & $\begin{array}{l}\text { Integrated engineering analysis, yield, } \\
\text { management, and production data min- } \\
\text { ing. } \\
\text { Off-line system to diagnosis control prob- } \\
\text { lems and target their APC investments. } \\
\text { Fab automation solution, including SPC/ } \\
\text { MES. }\end{array}$ \\
\hline Equipment Sector & $\begin{array}{l}\text { Detection, identification, and correction } \\
\text { of process and equipment faults. } \\
\text { Economic benefits and ROI in terms of } \\
\text { increased yield, fewer test wafers, fewer } \\
\text { scrapped wafers, and enhanced CoO. }\end{array}$ \\
\hline Controller Sector & $\begin{array}{l}\text { Control algorithm for low-running devices } \\
\text { using production data. } \\
\text { Standard sensor/tool communication and } \\
\text { fast data handling capability. }\end{array}$ \\
\hline Sensor Sector & $\begin{array}{l}\text { Automated system that monitors the } \\
\text { behavior of APC application. } \\
\text { Converting physical properties to process } \\
\text { information. }\end{array}$ \\
\hline
\end{tabular}

situ sensors have arisen as urgent issues to be resolved [1-4]. Five sectors that contribute to the APC technology and their roles are listed in the Table 1.

Among numerous semiconductor processes, plasma processing is one of the most important, with urgent requirements for real-time monitoring with advanced sensors. The monitoring and diagnosis of plasma etching started in the 1980s [5-7], and it is still a challenging topic in semiconductor process control. As allowable process margins continue to narrow, plasma monitoring techniques that include advanced in-situ process control and diagnosis are required [8]. Sensors considered for practical adoption in plasma etching are classified as electrical or optical ways, depending on their detection mechanism. Electrical sensors usually measure high-frequency RF signals in an RF matching network, and optical sensors measure the physical and chemical properties of the plasma glow discharge. Electrical sensors enable high-frequency data acquisition, but they sometimes require sophisticated hardware reconfiguration and calibration. Optical sensors can simply be mounted on the view port of the plasma chamber, but the interpretation of the optical spectroscopic signals is difficult without domain knowledge.

In this paper, the use of a chromatic monitoring method is presented for optical plasma process monitoring for the detection of process anomalies. Optical emission spectroscopy (OES), the most well-known plasma process monitoring sensor, provides high-dimensional plasma optical emission spectra, so dimensionality reduction or peak selection are always challenging. However, since the proposed optical monitoring system generates only three streams of red-green-blue (RGB) signals from a photo detector, high-speed optical data acquisition could be achieved that is as fast as current-voltage-type electrical sensors. Optical monitoring is non-invasive and is easy to install. Faster data acquisition enables the detection of extremely small perturbation, as short as hundreds of micro seconds. We report an observed abnormal plasma discharge as short as $210 \mu$ s.

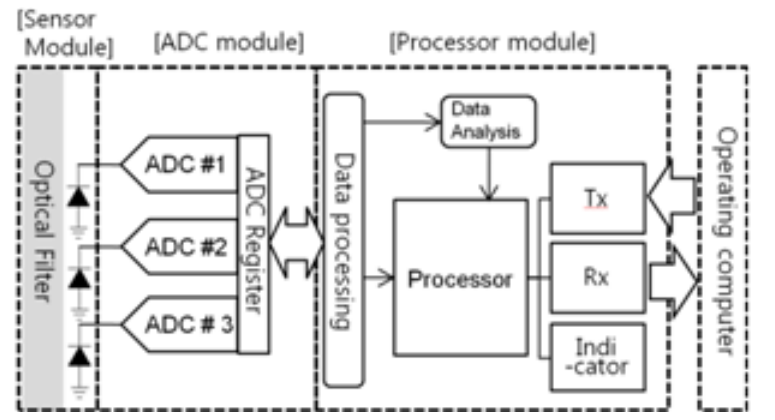

Fig. 1. System block diagram of the suggested optical real-time process monitoring sensor.

\section{SENSOR DEVELOPMENT AND EVALUATION}

\subsection{Sensor development}

Add-on sensors for process monitoring should be able to accurately identify any suspicious behavior at its onset. Chromatic monitoring in plasma processing as a non-invasive method was previously proposed by Russel et al. [9]. In their study, a chromatic monitoring system provided significant speed advantages over the equivalent spectroscopic technique, optical emission spectroscopy (OES). The Plasma Eyes Chromatic System (PECS) is designed to observe the optical emission of a plasma glow discharge, and represent it with red-green-blue (RGB) values. The peak wavelengths of RGB are $620 \mathrm{~nm}, 540 \mathrm{~nm}$, and $460 \mathrm{~nm}$, with response ranges of 590-720 nm, 480-600 nm, and 400-540 nm, respectively. A schematic of the hardware configuration is presented in Fig. 1. Data collected in the master controller are converted from analog to digital, and the digitally processed signals are transferred to a PC for data visualization at up to $10 \mathrm{kHz}$, depending on the speed requirement in the application. The PECS is specifically designed to be compatible with a semiconductor manufacturing environment, and a graphical illustration of the in-situ sensor-based process monitoring employed is presented in Figure 2. The system satisfies major requirements of in-situ process sensors, including a lack of perturbation to the plasma during the process, high sensitivity for low-intensity glow discharge, high data acquisition speed for RF frequencies, and automation compatibility. The sensor set-up is identical to that of OES, through a sidewall viewport through an optical fiber cable, and it is designed to detect anomalous processes, identifies process shifts and drifts, and notifies operators with an alarm when a fault is detected.

\subsection{HSB response evaluation}

An initial experiment was performed to test the sensor response and correlate the optical data with process conditions. By modifying the process tool parameters, we investigated the relationship between the in-situ optical sensor signals in response to the change in the process conditions (i.e., RF power, chamber pressure, and gas flow). A MINIPLASMA Station 13.56-MHz RF powered CCP-RIE was employed with baseline parameters of 300 $\mathrm{W}$ of RF power, an oxygen gas flow of $20 \mathrm{sccm}$, and a 30-mTorr chamber pressure. Each process parameter was modified while the other parameters were set to their baseline level. Figure 3 presents the observed results. In etch, the hue of the plasma color is mostly determined by the gas species injected into the process chamber, and it can also be changed by by-product gas species. The saturation of the plasma color might become clear 


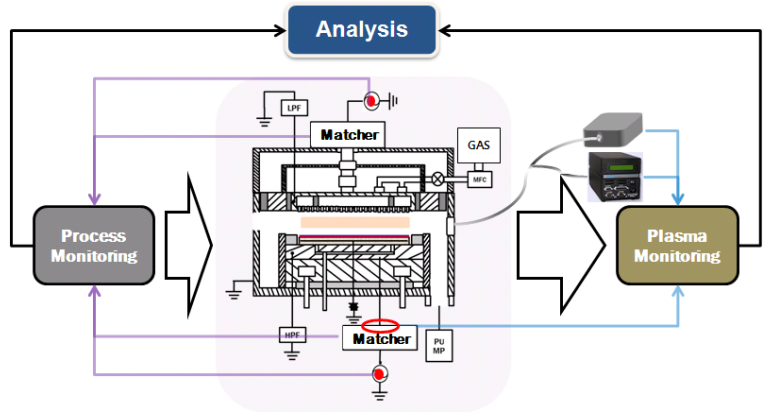

Fig. 2. An illustration of in-situ sensor-based process monitoring scheme.

under low chamber pressure. The brightness of the plasma color can also be varied depending on the amount of white components, and the combination of RGB is in white light emission.

Hue, saturation, and brightness (HSB) are aspects of color representation with red, green, and blue (RGB). All possible colors can be specified according to HSB, similarly to RGB, and visible light contains several light sources, whose energies are shown over a band of wavelengths. Hue $(\mathrm{H})$ is the wavelength within the visible-light spectrum at which the energy output from a source is greatest. Saturation (S) is an expression for the relative bandwidth of the visible output from a light source, and it can be found by the size of the wavelength band of the light sources. As saturation increases, color appears more "pure," and as it decreases, color appears more "washed-out." Brightness is a relative expression of the intensity of the energy output of a visible light source. It can be expressed as a total energy value or as the amplitude at the wavelength where the intensity is great. Given a color defined by $(R, G, B)$, where $R, G$, and $B$ are between 0.0 and $1.0,(H, S, B)$ are determined by the following equations:

$$
\begin{aligned}
& H= \begin{cases}\left(0+\frac{G-B}{M a x-M i n}\right) \times 60, & \text { if } R=\text { Max } \\
\left(2+\frac{B-R}{M a x-M i n}\right) \times 60, & \text { if } G=\text { Max } \\
\left(4+\frac{R-G}{M a x-\text { Min }}\right) \times 60, & \text { if } B=\text { Max }\end{cases} \\
& S=\frac{\text { Max }- \text { Min }}{\text { Max }} \text { and } B=\operatorname{Max} \times 100
\end{aligned}
$$

where Max and Min are the maximum and minimum values of $(R$, $G, B)$.

In Fig. 3, no obvious trends were found in hue and saturation, but a significant correlation was shown in brightness in response to changes in RF power, pressure, and gas flow. This experiment was performed without a wafer loaded in the chamber, so no byproduct would be observed. Thus, hue and saturation were not drastically changed. However, change in the process parameters affected the brightness of the detected optical emission. The increased RF power shown in Figure 3(a) increased the atomic/ molecular collision of plasma to generate higher optical intensities of all the sources of light. In the same manner, Fig. 3(b) reflects the increased possibility of atomic collisions from the decreased mean free path of the molecules in plasma. A monotonic decrease in brightness is interpreted as decreased total photon emission energy observed by the optical detector. When the chamber pressure is maintained at a certain level, the increased amount of oxygen gas will intensify the oxygen plasma, the color
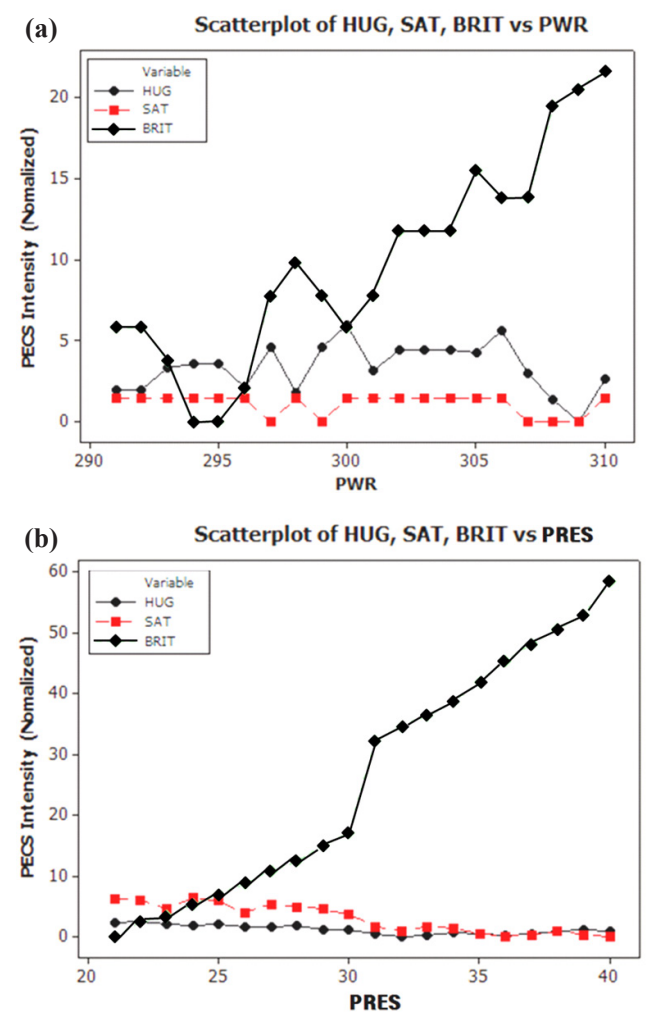

(c) Scatterplot of HUG, SAT, BRIT vs Gas (02 sccm)

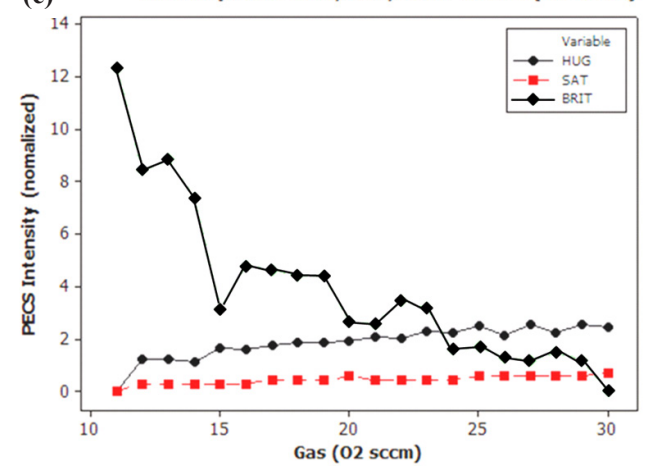

Fig. 3. Responses of optical emission intensity of PECS with respect to the variations of (a) power, (b) pressure, and (c) gas flow of oxygen.

Table 2. Baseline process and ranges of variation.

\begin{tabular}{lccc}
\hline \multicolumn{1}{c}{ Parameters } & Baseline & Range & Step \\
\hline \hline Pressure (mTorr) & 100 & $90-110$ & 5 \\
\hline Top power (watt) & 400 & $300-500$ & 50 \\
\hline Bottom power (watt) & 400 & $300-500$ & 50 \\
\hline$C_{4} F_{8}(\mathrm{sccm})$ & 10 & $8-12$ & 1 \\
\hline $\mathrm{CH}_{2} F_{2}(\mathrm{sccm})$ & 12 & $10-14$ & 1 \\
\hline
\end{tabular}

of which is white, and it causes a reduction in its brightness. Therefore, the suggested optical monitoring method is useful for monitoring the change in plasma process conditions.

\subsection{In-Situ monitoring}

Employing an eight-inch $(200 \mathrm{~mm})$ production etching tool, five consecutive blanket oxide etch runs were performed while monitoring the plasma glow discharge using PECS. The etching tool used was an Exelan ${ }^{\mathrm{TM}}$ dielectric etcher manufactured 
Table 3. Process fault scenario for the blind test.

\begin{tabular}{llc}
\hline Run No & \multicolumn{1}{c}{ Recipe } & Type \\
\hline \hline Run \#1 & Baseline (No disturbance) & Baseline \\
\hline Run \#2 & $\begin{array}{l}\text { During normal process run, bring Top Power dis- } \\
\text { turbance within +/ - 5 watt variation. }\end{array}$ & Abnormal \\
\hline Run \#3 & $\begin{array}{l}\text { During normal process run, bring Pressure distur- } \\
\text { bance, within +/-2 mTorr variation. }\end{array}$ & Abnormal \\
\hline Run \#4 & $\begin{array}{l}\text { During normal process run, bring } \mathrm{C}_{4} \mathrm{~F}_{8} \text { Gas distur- } \\
\text { bance, within +/- 1 sccm variation }\end{array}$ & Abnormal \\
\hline Run \#5 & Comparison run (No disturbance) & Baseline \\
\hline
\end{tabular}

by Ram Research. The baseline process parameters and ranges are provided in Table 2. Each run lasted for one minute, and the process parameters were gradually modified every 10 seconds to quantitatively correlate the sensor response to process conditions.

In Figs. 4(a)-(e), the sensor response is presented with respect to the process parameter modifications. As soon as the plasma was ignited, the optical response reflected the complicated plasma dynamics of dielectric breakdown in the gas phase. When the process was stabilized, the optical intensities measured by PECS followed the change in process parameters. For instance, when the pressure was increased (Fig. 4(a)), the sensor reading decreased. Absolute quantification of the pressure cannot be directly measured by PECS, but the relative deviation from the baseline can be quantified. The effect of power variation is also quite clear, as shown in Fig. 4(b).

However, more surprising was the fact that the optical sensor could detect changes in gas flow, as shown in Figs. 4 (c) and (d). Comparing these experimental results to the pressure and RF power variation shown in Figs. 4 (a) and (b), the acquired optical signals from the gas flow tracked the variation less accurately. The results seem to indicate that optical monitoring is less sensitive to gas flow compared with other process variables, but the rationale for slow tracking originated from the physical delay of gas delivery to the chamber. When the gas setting changes, there is an inevitable delay for the gas to be delivered to the chamber until the change is reflected in the plasma glow discharge. The delay depends on the length of the gas delivery line between the mass flow controller and the process chamber. Therefore, this optical signal is in fact also sensitive to the change in plasma glow discharge induced by the amount of gas flow change.

\subsection{Blind test}

Following the affirming results presented above, we extended the investigation to explore a more realistic scenario for a manufacturing environment by examining small perturbations in process parameters. This experiment was performed with the same 200-mm tool, but the process parameters were not provided to us until the test was reported to the participating engineer (thus, a blind test). Five consecutive blanket oxide etch runs were performed while the plasma was monitored with a sampling rate of $1 \mathrm{~Hz}$. The first and the last runs used the baseline process parameters to compare the absolute optical intensity levels to examine repeatability. The other runs were regarded as abnormal in power, pressure, or gas flow. The result is presented in Figure 5. To simulate process shifts, the individual parameters of interests were perturbed from the baseline values. For the first 40 seconds of each run, the parameters remained at the baseline, and then four arbitrary perturbations were applied individually every 15 seconds. Finally, the modified parameters were returned to their
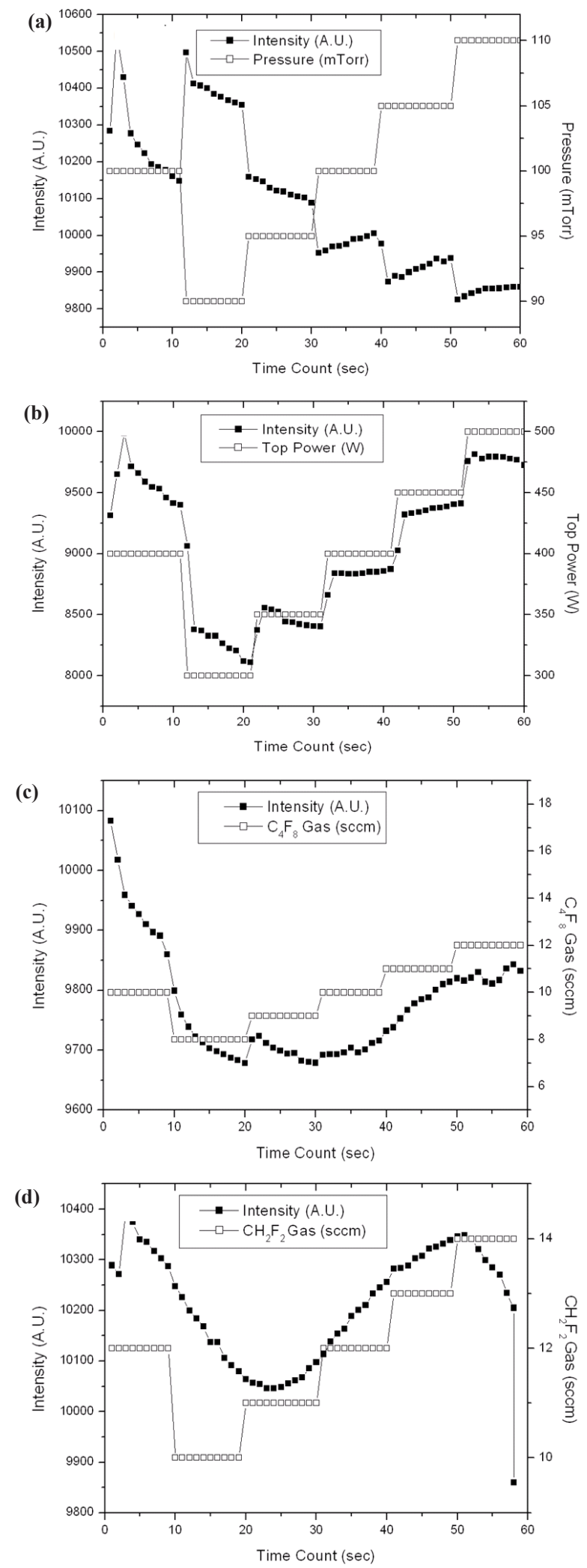

Fig. 4. Optical emission intensity reading of PECS w.r.t. the process parameter variations, as presented in Table 2.

baseline level for the remaining 20 seconds. In Fig. 5(a), of the baseline RF power was set to 400 watts, and perturbations of $\pm 2.5 \%$ and $\pm 1.25 \%$ were applied. For gas flow perturbation, $\pm 1-2$ sccm of $C_{4} F_{8}$ was applied to a baseline level of $10 \mathrm{sccm}$, and the 

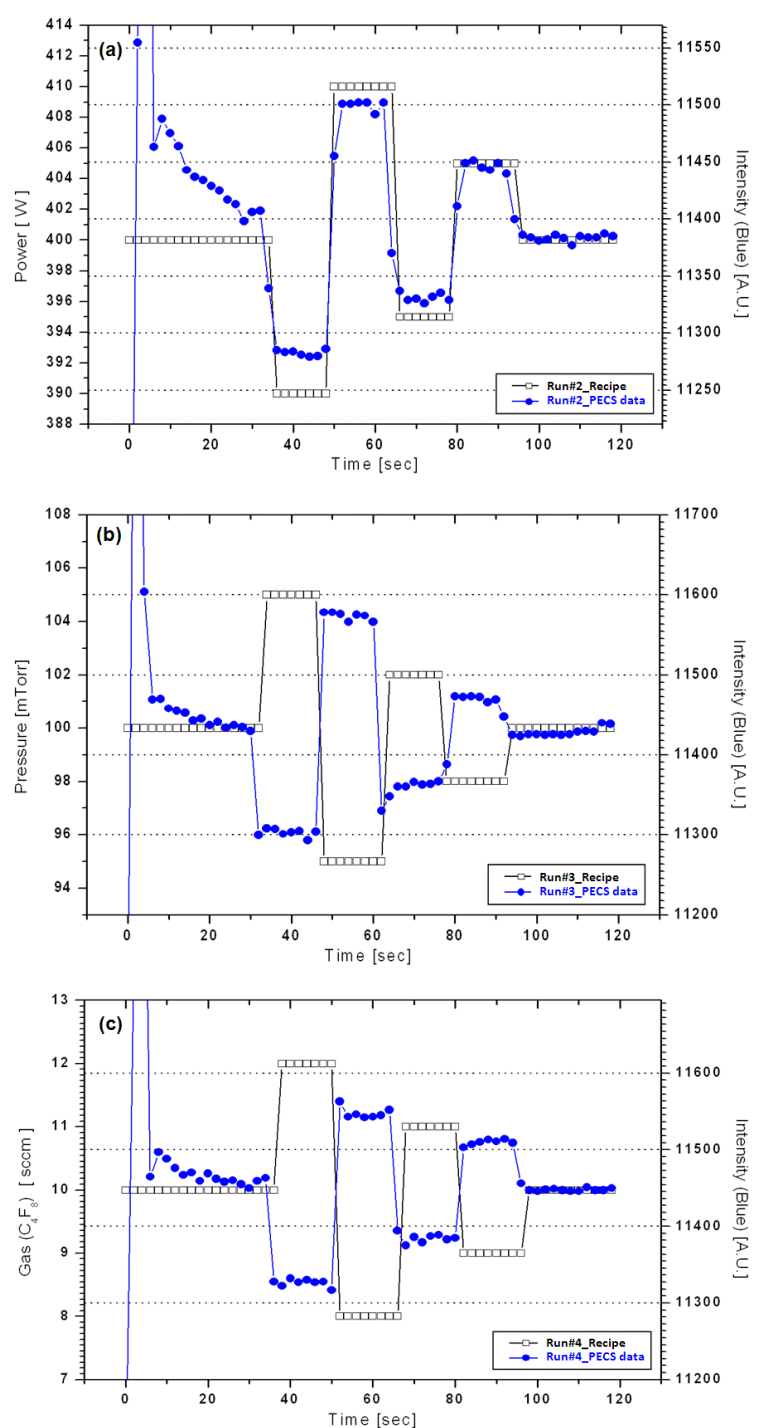

Fig. 5. Blind test result for the evaluation of the proposed sensor response.

results are shown in Fig. 5(c). From the figures, it is evident that small perturbations of RF power, pressure, and gas flow change the state of plasma glow discharge. This can be detected by the PECS sensor, which can clearly serve as a signal indicator for detecting a faulty process.

\section{PLASMA ARC DETECTION}

It has been reported recently that plasma chamber arcing issues are of significant concern. Anomalous plasma discharge resulting in arcing is not yet monitored systematically, but it can make plasma conditions unstable, generate unexpected particles during the process, cause electrical damage to devices when the arc hits a metal contact opening or metal pattern in subsequent layers, burn or break wafers, or damage the electrostatic chuck in the etching system. One hypothesis for the cause of plasma arcing is the accumulated or unbalanced local discharge between the chamber and plasma or between the wafer and plasma. Noticeable arcs, called macro-arcs, can be easily detected in the form of sparks during the process or lightning marks after the process. Once this kind of arc takes place, the chamber must be

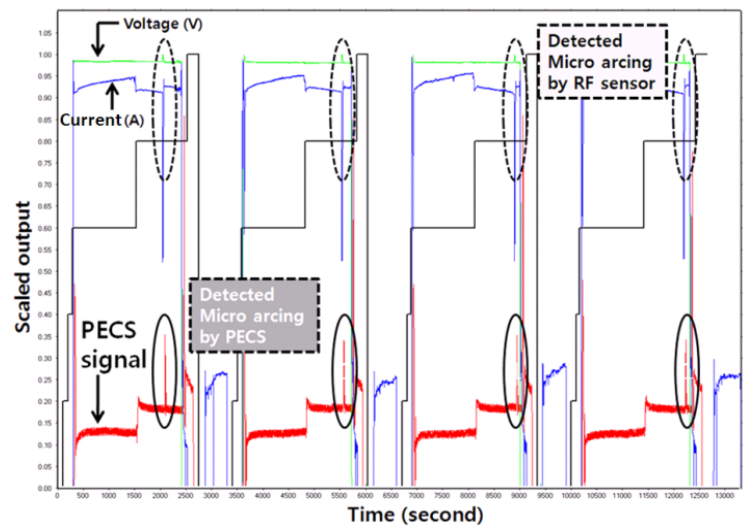

Fig. 6. Real-time detected plasma arcing inside the process chamber.

cleaned for the next process. However, we postulate that such a macro-arc is caused not by a single event, but by the accumulation of undetected micro-arcs. To avoid catastrophic failures that might cause wafer damage or unscheduled equipment maintenance, detecting micro-arcing during the process in real-time is crucial.

Previously, RF I-V probes have been suggested for arc detection, but such devices require hardware reconfiguration for the attachment of the sensor to the plasma tool. The specific mechanism of micro-arcing is still unknown, but it has been reported that plasma arcing is related to decreased electron density and increased electron temperature [10]. A practical approach to arc detection in a production environment is required, and we believe that this can be accomplished by detecting changes in plasma characteristics. Non-invasiveness is a high priority for real-time monitoring. In addition, to realize timely and accurate detection of arc events with durations of less than tens of microsecond, we propose the use of the PECS, a plasma monitoring system with $10-\mathrm{kHz}$ data sampling rate capability. This frequency of data acquisition enables the detection of arcs of less than 10ms duration, and optical monitoring via sidewall viewports in the etching system alleviate any concerns regarding intrusiveness and perturbation of the plasma or RF power that can result from the use of an external I-V probe.

The experimental exploration of this approach consisted of 900 consecutive runs without a significant interrupt other than a prescheduled tuning and minor maintenance. Patterned silicon oxide wafers were etched in a $300-\mathrm{mm}$ cluster tool for a production qualification test. An OES system and PECS were employed for in-situ plasma monitoring and arc detection, respectively. In addition, a commercially available RF detector was simultaneously used for monitoring any anomalous plasma arcs during the test.

One of the test recipes consisted of a harsh environmental test, so that the possibility of chamber arcing was expected to be higher than under normal operation. As shown in Fig. 6, micro arcs were successfully detected in both the electrical and optical sensors. The amount of change in the electrical signal appeared to be significant, but the optical signal observed by PECS was more clearly noticeable. Detection occurred at identical points in both systems. While analyzing the detected arcs in Fig. 6, intensive discussions with process engineers were conducted to determine whether the signals represented true or false alarms. We have successfully detected several arcs during marathon tests, and other examples of detected arcs are presented in Fig. 7. Figure 7(a) shows a typical example for a relatively extended arc, and Fig. 7(b) shows the shortest arc that we observed. 

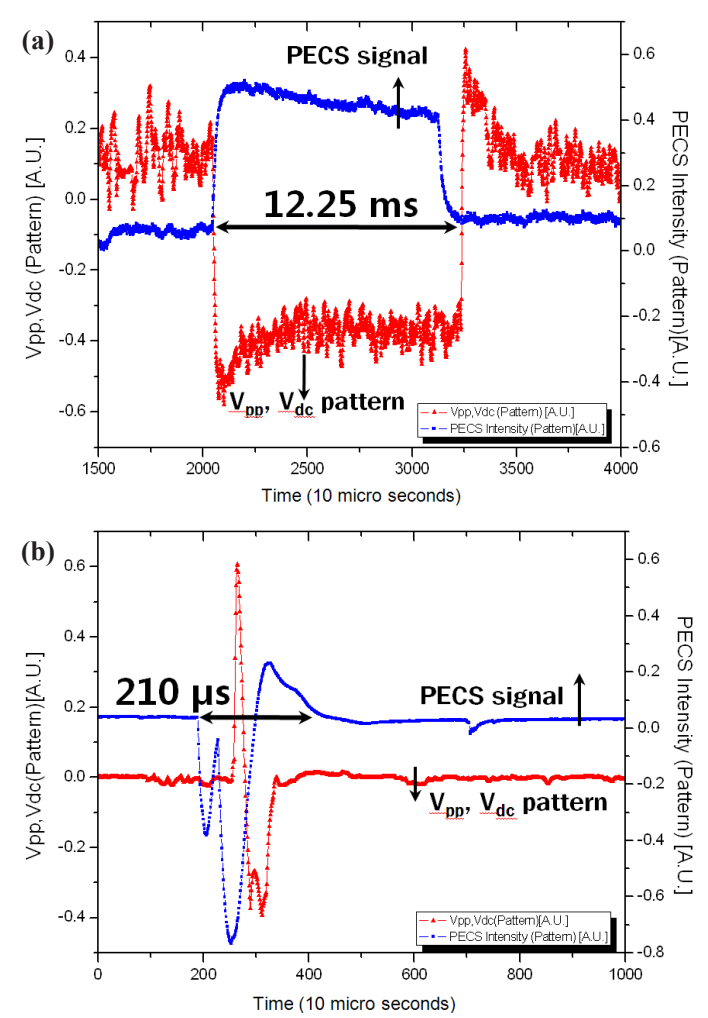

Fig. 7. Optically detected arcs in comparison with high frequency electrical signals.

\section{LONG-TERM REAL-TIME PROCESS MONITORING}

While developing a next-generation device fabrication process for high throughput and low plasma charging damage, realtime in-situ monitoring of abnormal discharge in plasma was the most critical factor to consider. To alleviate the concerns of abnormal plasma discharge in a new process development, a marathon wafer running for more than a month was performed with three types of in-situ monitoring add-on sensors installed, including optical emission spectroscopy, a high-frequency IV probe, and PECS. During the marathon test, several interesting phenomena regarding abnormal plasma discharge. When the magnitude of abnormal discharge was sufficiently large, it was easily detected by tool data associated with the RF matcher, such as the capacitor position and reflected RF power. When accumulated charge is abnormally discharged into plasma, the discharge became additional current source in the plasma. Thus, the RF matching network detects this instantaneously increased current in the plasma, and it adjusts the impedance matching by modifying the rotating capacitor positions in the matcher. The RF IV probe connected between the RF matcher and electrode also observes the abnormal discharge in the same manner, and it generates significant current drop within a very short period. Figure 8 shows how the IV probe detected this abnormal discharge.

Simultaneous comparison of optical monitoring with the electrically detected abnormal discharge is presented in Figure 9. The abnormal discharge detected on 08/19/09 at 09:26:03 is shown in Figs. 8 and 9, but the suggested optical monitoring method observed the abnormal discharge or its symptoms in three wafers in advance comparing with electrical measurement. We also investigated tool data of the bottom electrode. Figure 10 (a) shows that no significant anomaly was observed in the Bot-

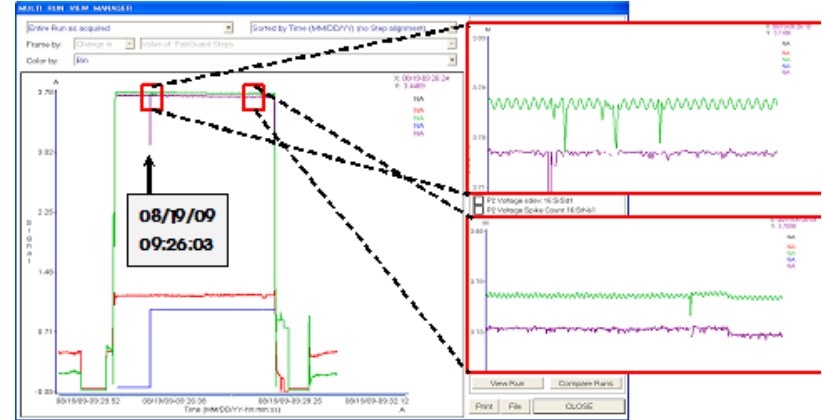

Fig. 8. Detection of abnormal discharge by add-on IV probe during a marathon test.

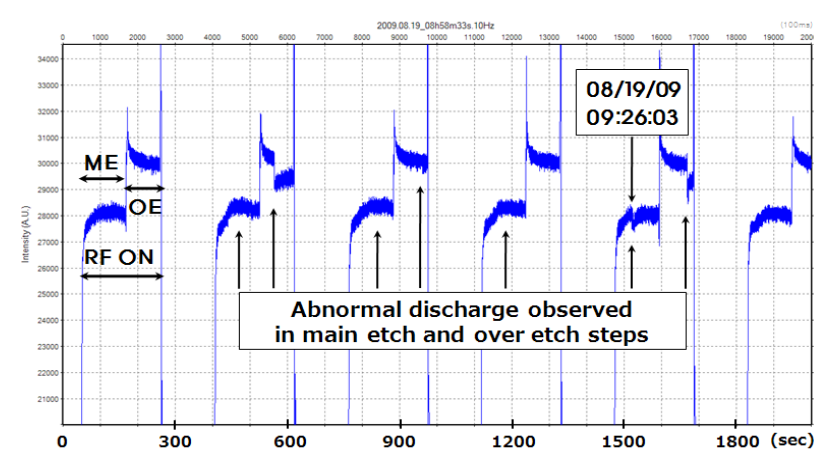

Fig. 9. Detection of abnormal discharge by PECS during a marathon test.
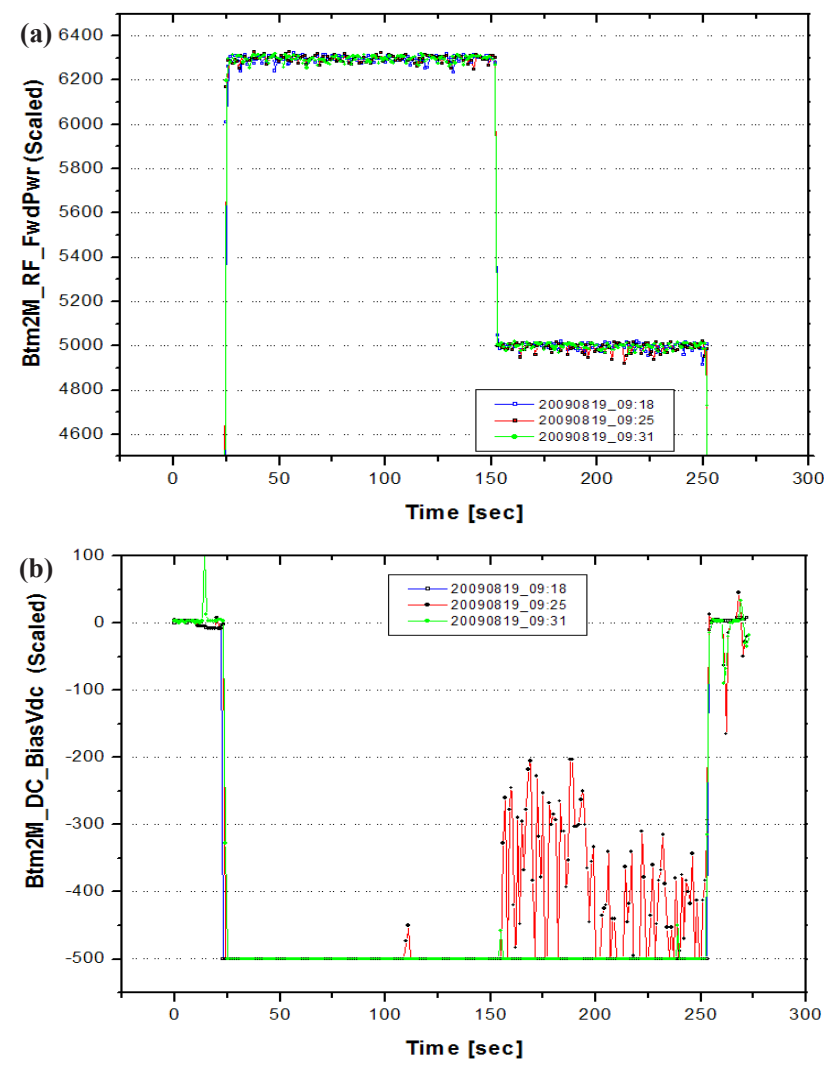

Fig. 10. Tool data observation at bottom electrode: (a) $2 \mathrm{MHz}$ RF forward bias and (b) DC bias. 
tom RF forward power in the corresponding three consecutive runs. However, the DC bias measured at the bottom electrode successfully detected such abnormal discharge. We postulate that the charge that continuously accumulated in the chamber was suddenly discharged by the accumulated electrical potential. When it was discharged onto the wafer or DC biased wafer chuck, it became noticeable at the bottom electrode, as shown in Fig. 10(b).

\section{CONCLUSION AND FUTURE WORK}

The PECS optical plasma process monitoring system has been introduced, and its application as a signal indicator for realtime process fault detection and as an arc detection system was explored. The development, verification, and adoption phase for the sensor system have been presented. The response of the PECS system was evaluated by modifying the process parameters (including RF power, chamber pressure, and gas flow) and observing the sensor behavior. PECS proved to be very sensitive to RF power changes, even allowing their quantification to some degree. Anomalies induced by the chamber pressure and gas flow variations were also observed. The system was also evaluated as an arc detection mechanism with promising results. Overall, the proposed PECS possesses great potential as a multi-purpose insitu sensor for realizing sensor-based plasma process control. The remaining task for the full validation of the PECS system is integration within a control system framework. The combination of in-situ process monitoring data with fault detection and classification systems will be of great value in achieving the ultimate objective of real-time advanced process control (APC).

\section{ACKNOWLEDGMENTS}

This work was supported by the Semiconductor Process Diagnosis Research Center (SPDRC) at Myongji University, and the Electrical and Computer Engineering Fellowship Program at the
Georgia Institute of Technology. The authors are grateful to the National Nano Fab Center for performing the sensor verification and to Hwabaek Engineering for their technical support.

\section{REFERENCES}

[1] J. Moyne, H. Haji, K. Beatty, and R. Lewandowski, IEEE Trans. Semi. Manufac., 30, 408 (2007) [DOI: http://dx.doi.org/10.1109/ TSM.2007.907617].

[2] S. J. Qin, G. Cherry, R. Good, J. Wang, and C. A. Harrison, J. of Process Control, 16, 171 (2006) [DOI: http://dx.doi. org/10.1016/j.jprocont.2005.06.002].

[3] P. Kang, H. Lee, S. Cho, D. Kim, J. Park, C. Park, and S. Doh, Expert Systems with Applications, 39, 12554 (2009) [DOI: http:// dx.doi.org/10.1016/j.eswa.2009.05.053].

[4] Y. Kim, K. Baek, S. Lee, S. Lee, J. Lee, Y. Jee, C. Kang, H. Cho, and J. Moon, Int. Symp. Semi. Manufac., 346 (2006) [DOI: http:// dx.doi.org/10.1109/ISSM.2006.4493103].

[5] S. Dolins, A. Srivastave, and B. Flinchbaugh, IEEE Trans. Semi. Manufac., 1, 23 (1988) [DOI: http://dx.doi. org/10.1109/66.4369].

[6] G. May and C. Spanos, IEEE Trans. Semi. Manufac., 6, 28 (1993) [DOI: http://dx.doi.org/10.1109/66.210656].

[7] S. Hong and G. May, "Neural Network-based Real-time Malfunction Diagnosis of Reactive Ion Etching Using in-situ Metrology Data," IEEE Trans. Semi. Manufac., 17, 408 (2004) [DOI: http://dx.doi.org/10.1109/TSM.2004.831952].

[8] C. J. Kang and S. C. Park, "Semiconductor Patterning Technology for Nano Devices,” Current Applied Physics, 8, 656 (2008) [DOI: http://dx.doi.org/10.1016/j.cap.2007.04.059].

[9] P. C. Russel, I. Khandaker, E. Glavas, D. Alston, R.V. Smith, and G.R. Jones, IEE Proc. Sci. Meas. Technol., 141, 99 (1994) [DOI: http://dx.doi.org/10.1049/ip-smt:19949850].

[10] M. Sato, H. Ohtake, K. Suzuki, S. Samukawa, J. Vac. Sci. \& Tech. A: Vacuum, Surface, and Films, 25, 1594 (2007) [DOI: http:// dx.doi.org/10.1116/1.2796180]. 